\title{
A study on ESL vocabulary acquisition needs and classroom practice: a Bangladeshi context
}

\author{
SAYMA ARJU
}

\begin{abstract}
The main objective of teaching vocabulary in EFL classes is to empower the learners with sufficient word stock for successful communication. Observations show, for both EFL instructors and learners (of Bangladesh), vocabulary acquisition is an important but tiring task as in most of the cases learners need to memorize blindly lengthy word lists suggested by their instructors as a practice of vocabulary learning. But in their real life communication, learners could rarely use any word that they have been taught as vocabulary items in language classes because only dependency on memorization cannot serve them properly with the right word/s at times of their needs. This study critically analyzes the reasons that makes vocabulary related tasks done in EFL classes mostly unsuccessful and suggests some recommendations that could come in help for achieving an improved outcome. Though the study is done focusing the context of Bangladesh, the picture is more or less common in all EFL contexts. Therefore, the recommendations are applicable anywhere where ESL / EFL Vocabulary acquisition is the concern.
\end{abstract}

\section{Introduction:}

"Many students consider learning vocabulary (as) a tedious job . . . (and) start blaming their poor memory . . . They come to rely on incidental learning; finding individual studying boring and insufficient" (Gnoinska, 1998, p.12). It has also been affirmed by a number of scholars that learners learn even by picking vocabulary from their own world. That may have happened from watching their favourite cartoon show to interaction in and outside the language classroom and above all from classroom activities. Teaching vocabulary turns into an unavoidable issue of teaching a language as learners' receptive and comprehensive capability through sounds and symbols is quite dependent on the volume of vocabulary they are possessing. Experiences of yesteryears are indicating that in general ESL learners of 
Bangladesh failed to obtain a satisfactory competence in using English and their insufficient stock of vocabulary has been detected as one of the underlining reasons. In order to have a glimpse of the techniques and methods practised at present in teaching vocabulary, data collected from learners of different grades, teachers, learners' class work copies, university test papers etc. found that the problem lies in planning, presenting, practising and evaluation policies that are involved in teaching this micro skill. The aim of the paper is to point out the inconsistency between the target and techniques that are used to achieve the target. Here, the word "target" implies the minimum stock of vocabulary needed for continuing fluent communication. Besides, some recommendations are proposed to minimize the specific problems.

\section{Why vocabulary learning is so essential for ESL Learners:}

While commenting on the ESL learners' competence in speaking and writing, language instructors frequently complain that the learners are lagging behind because of having an inadequate stock of vocabulary. Dong (2009), in "Beyond Reading: Vocabulary Expansion and Language Use Consolidation" points out, "Vocabulary is an important part of reading. Without sufficient vocabulary, readers may make a wild guess at the unknown words, but are unable to fully understand the reading texts, or just understand them vaguely, and many a time wrongly." (Introductory section, para.3). Besides, learners' comprehensive ability is quite dependent on their knowledge about vocabulary. It is assumed that as much vocabulary as stock the learners possess, they will show that much confidence in handling the language. Though for Stevick (1976) "language is a social process . . . (and) language arises in the life of individual through an ongoing exchange of meaning with significant others" (p.160), improving vocabulary is one of the objectives of EFL classes as this activity gradually helps the learners in achieving motivation, self-confidence and proficiency. 


\section{Theories about Vocabulary Learning:}

Theories about vocabulary learning could help ESL instructors to design activities for getting maximum output from vocabulary related task done in the classroom. To quote from Richard (1985):

knowing a word means knowing the degree of probability of encountering that word in speech or print. For many words we also "know" the sort of words most likely to be found associated with the words . . . Knowing a word implies knowing the limitations imposed on the use of the word according to variations of function and situation ... Knowing a word means knowing the syntactic behavior associated with that word . . . (that) entails knowledge of the underlying form of that word and the derivations that can be made from it . .. (and) knowledge of the network of associations between that word and other words in the language ... (and) knowing the semantic value of a word ... (and) knowing many of the different meanings associate with the word. Many students have not been able to acquire such in-depth knowledge of the words they encounter, and therefore fail in subsequent reading tasks or language use. Various kinds of post-reading tasks can strengthen the memory of the vocabulary they have learned, expand the use of the learned language, and effectively help solve the problems afore-mentioned. (Pp.178-82)

For Hulsjin, as cited in Ghazal (2010), preparing learners with strategies necessary to expand their vocabulary knowledge could be one of the targets of teaching vocabulary. Though the strategy has a basic shape, different learners handle it in different ways in terms of their age, motivation and training. $\mathrm{Gu}$ and Johnsons (1996), consider ESL vocabulary learning as the following chain in "Vocabulary Learning Strategies and Language Learning Outcomes":

Meta cognitive $\rightarrow$ Cognitive $\rightarrow$ Building up memory $\rightarrow$ Activation

Meta cognitive stage employs careful selection of important vocabulary from a source. Adult learners could choose words from a source by being self motivated while for the young learners, it is done by the instructor. Cognitive strategy means getting the meaning of particular word/s and phrases. This strategy may involve 
learners either to look at the dictionary or to guess an idea about a new word or phrase after a minute observation of surrounding words with an assistance of grammatical clues and background knowledge. Building up memory requires repetitions and rehearsals. This strategy involves successful encoding ability of the learnt vocabulary by identifying, associating and contextualizing. And in the activation level learners become experts to use their vocabulary knowledge for real life communication.

On the other hand Nation (2001) proposes vocabulary learning activities as a consecutive process of "planning", "source" and "processes". Planning involves identifying and selecting words, strategy of presenting and repetitions. Stage known as "Source" involves activities for getting information about a word both contextual and literal. The last category of Nation's taxonomy of vocabulary learning strategies is a process that includes establishing word knowledge through notices, retrieving and generating strategies. Noticing involves identifying, retrieving involves recalling and generating is a strategy that helps to use the word in new context. Therefore keeping the strategies in mind, a standard vocabulary learning lesson could explicitly be comprised in the following steps:

1. Setting the context

2. Eliciting vocabulary

3. Drilling

4. Asking relevant Concept Check Questions

5. Practice activity

But getting a satisfactory result from using any of the strategies could be possible only if the learners are convinced that the strategy really will come to their benefit (Ellis,1994), and be trained for using the strategy independently. Ghazel (2010) puts importance on vocabulary learning strategy training saying that learners should be trained and motivated to enhance their vocabulary by listening to the radio, watching movies, and indulging in self study. 


\section{The objective of teaching vocabulary in Bangladesh:}

The Education Ministry of the People's Republic of Bangladesh has undertaken an incentive care for learning English. According to the curriculum of National Curriculum of Textbook Board (NCTB), English is taught from class-1 to the graduation level with an objective to provide the learners an opportunity to learn another language besides the mother tongue and is expected that the learners could achieve functional competence through this trial. It is worth mentioning here, for many students English for Today, the compulsory text book which is developed and supplied by the NCTB is the only book that they have ever found written in English. Considering the fact, this series has attempted introducing a number of new vocabulary items to support and carry on the language activities. For example, English for Today designed for class-viii contains about 140 new words in its 75 lessons. Surely the number of new vocabularies is increased in the books for senior grades eg. book maps' data show, in English for Today designed for class ix $-\mathrm{x}$ the number of new words is 514 and in English for Today designed for class xi-xii is 835 . It is worth mentioning here that about $95 \%$ of the enlisted vocabulary items are individual words and the other $5 \%$ contains either phrases or clipped words. However, new items are often found in consecutive lessons with a purpose of reinforcing and highlighting their usages in different contexts, though a few exercises of the books demand the newly presented words to solve.

The existing patterns of public examinations' questions are also planned to evaluate the learners' competence to understand English vocabulary. The model question (for English First paper) provided by the NCTB for the SSC (Secondary School Examination) allots about $25 \%$ of the total marks for testing the knowledge of vocabulary. It is to be noted here that in Bangladesh any one can chose either NCTB's curriculum (English/Bengali medium in instruction) or British Curriculum of GSC O' Level and A' level until the higher secondary education. English medium schools that follow the British Curriculum and prepare learners for GSC O' Level or $\mathrm{A}^{\prime}$ level give more importance in teaching vocabulary. Learners need to learn new words encountered in each lesson in every subject. Again, the medium of instruction of the graduation level in most of the universities is English. Here learners of any discipline must undergo some general English courses though the 
number of courses varies. As teaching English language without teaching vocabulary is impossible, special vocabulary items related with the courses are taught even in the tertiary level. These courses are designed to prepare learners for taking the challenges of understanding an advanced reading material as well as to achieve an ability to write in standard English to meet professional requirements.

Studies show that for TESL learners' vocabulary profiles and academic performance are co-related (Cobb and Morries, 2004). In case of Bangladeshi students situation proved alarming when about $80 \%$ candidates show poor performance in English in the university admission tests. Concerning the purpose of the paper a close observation has been made on answer scripts of 100 candidates of such an admission test taken by a private university of Dhaka City on May,2009. A special attention was given to the writing part where candidates were asked to write a paragraph on a fixed topic. From these answer scripts at least three weak points regarding vocabulary were identified: (i) common errors caused by misunderstanding the meaning of words or phrases; (ii) choosing wrong word/s or synonym; (iii) little variation in selecting vocabulary for comprehension etc. Fallahzaden (2007) stated that using of a bilingual dictionary is one of the reasons for choosing wrong synonyms while Muncie (2002) found (as stated in Fallazaden) that limited stock of vocabulary is a 'major obstacle to students' learning to write in a foreign language. He (points out) that vocabulary learning is very important to the development of ESL writing and that ESL writing instructors need to recognize and encourage vocabulary learning.

\section{Present Practice of Teaching Vocabulary in Bangladesh:}

Later to form a clear view about the present practice of teaching and learning vocabulary in Bangladesh a series of surveys have been done. As its first step a general survey was done on 20 students of different grades asking the following questions: 


\section{Questionnaire -1}

a) Do you want to enhance the stock of the vocabulary that you have now? Yes/ No

b) Do you enjoy learning vocabulary as a part of the class work? Yes/ No

c) Why do you /don't you enjoy it?

While answering question (a) 100\% percent participants replied in the affirmative, but answers for question (b) showed the opposite reaction. Findings discover that the reasons for showing disinterest (of learners) in vocabulary learning or related tasks, lies in the following mentioned issues in particular-

- Learning vocabulary is a tiring task,

- Teachers always give a long list of vocabulary,

- Though they read a lot and try to memorize the word list, they easily forget either the word at times of need or the meaning of them while answering in the examination

Next task was done with the students' English Literature or English -1 class work copies of class KG-1 to class- VIII. These copies are collected from six different schools of Dhaka city. Among them two samples were taken from two leading English Medium Schools that follow the British Curriculum, two were from English version schools that follow the national curriculum and use English as the medium of instruction (known as English Version Schools) and the other two were from Bengali Medium schools that follow the national curriculum and use Bengali as the medium of instruction. These students' copies show that the English Medium schools use Radiant Reading series for teaching English Literature. Their general activities of teaching vocabulary are as follows: learners are asked to memorize 1020 words with meaning from each story; words (some other) are given for makesentence exercises; occasionally the learners practise finding out rhyming words etc. Among these word lists rarely there are phrases, compound or clipped words. In the English Version schools vocabulary are taught in the same manner though 
they use English for Today, Radiant Reading series and Oxford Reading Circle as text books for English Literature. Naturally in this case the word list becomes longer and the poor learners need memorizing it. Soon frustrations seize the learners as in the mean time they have experiences in forgetting either the meanings or the words during tests. Thus, vocabulary learning appears to them as a fearful task. Whereas in Bengali medium schools no vocabulary learning task is done from English for Today, but a series of words (these words are chosen according to alphabetical order without keeping any other correlation among them) are taught from the dictionary as a part of their class work, though no question is set to test this activity. Therefore, learners pay less attention to the task. To answer a question like "How do the learners manage to answer the vocabulary based exercises that usually appear in board questions?" both the teachers and learners replied that for this they usually solve fill in the blanks exercises given in different guide books available in the market and SSC Test Papers etc. Consequently, when these learners come to universities, for most of them securing a good score in language courses becomes a troublesome task because in this case the practice of memorization is unable to help them at all. However, findings of a survey (done on the 50 learners of under graduation level) show that in the grammar based courses, learners are not asked to learn any vocabulary for taking examination preparations though some of them claim that their instructors have given them some web sites' addresses and asked them to practice vocabulary related exercises by taking help from there as a part of their self study. But in the course like "Certificate in English Language Proficiency", "Fundamentals of English-2" or "Composition" importance on learning vocabulary is emphasized as a part of reading comprehensions, vocabulary building exercises are practised. But, for these courses most of the instructors prefer to keep the tasks limited to cloze test activities (like fill in the blanks and multiple choice exercises). Interestingly, the learners also argued that the vocabulary learning practices done in the class could help them a little in language tests because for them, the reading comprehensions that appear in the question papers are matters of surprises and hardly any word that they have practised in the classes appeared either in the reading passage or in the exercise followed by it. Here as well the goal of teaching vocabulary failed because learners need to depend on their blind guesses for answering such tests. 
The students' copies give an account of the manner of selections (choosing word, way of presentation, meanings etc.) related with teaching vocabulary. It has been seen that teachers' version of word meaning and explanations seems more complicated than the original one. It is because, as assumed from the students' copies, for presenting new vocabulary teachers preferred to go for the dictionary meaning especially the first meaning as arranged in the Oxford Learners' Dictionary whereas sometimes a synonym or an image or an example could serve the purpose. Besides, this strategy of choosing meaning sometimes takes the word away from its contextual meaning. But above all, the most harmful thing is to go for an incomplete meaning. Some examples of such activities (collected from the students' copy) are shown below:

Spade: a garden tool with a long wooden handle and a large flat blade made of iron

Trap: to catch the animal

Bison: a large, wild animal like a cow with long hair

Vine yard: the area of a land

Bruise: to cause an injury to some part of the body and make feel unhappy and less confident

Acrobat: person who can do clever things with his body

Any vocabulary item needs to be judged under the scale of frequency, availability and the learners' communicative needs before selecting it to be presented in the ESL vocabulary lesson. To learn about the typical practice of selecting important vocabulary from reading passages, data were collected from 40 teachers who teach English in secondary schools in different parts of Bangladesh. In groups they were asked to-

- Select important vocabulary items for the text

- Find out some suitable ways for presenting words/phrases that they have selected 
- Find out suitable tasks to be carried out as classroom activities on vocabulary

Teachers were informed beforehand that they would prepare a lesson plan suitable for the lesson for the students of class-ii of English version schools. In practice, from a passage of 150 words they chose 25 words as important vocabulary items and as means of presentation they mainly went for dictionary meaning. Of course, on one or two occasions they used teaching aid like board drawing. Among the possible practice tasks "make sentence" activity was top listed.

Teachers' Work-Sheet: 1

Q-1: Think you are going to teach the following part of the story as a reading and vocabulary exercise. When will you prefer to teach vocabulary?

i. Before the lesson ii. After the lesson iii. Middle of the lesson

Q-2: Read the following part of the story. Select vocabulary items that you find important to teach the students of class -ii of English version schools. In your answer scripts write down the way of presenting each word.

Q-3: List suitable tasks for teaching the listed words.

Next work was done with another 30 senior teachers who had undergone at least a training session from any of the institutions/projects like: ELTIP (English Language Teaching Improvement Project), BRAC (Bangladesh Rural Advancement Committee), BIAM Foundation (Bangladesh Institute for Administration and Management) or TQI (Teaching Quality Improvement Project). At first, the Participants were given a few simple words (like- snake, strawberry, halves and celebrate) and asked to show the possible ways of presenting them. Participants made a satisfactory response as their presentations were supported by flash cards, board drawing and realia though some of them were confused about the idea of using model and realia. But when this group was asked to do the same work with a lesson from English for Today: grade-vii, they made a disaster. In their demonstration the participants really did a mess: some of them just went for identification of the new words of the lesson without a suitable explanation; some 
other faced problems in presenting abstract words, but none of them concentrated on the suitable activities for practising and reinforcing the newly learnt items. The very session was followed by a training session on vocabulary presentation and practice and later by another demonstration. In this training, participants were also informed about-

- the ideas that vocabulary teaching includes (e.g. Presenting new words, Making passive vocabulary in active vocabulary, some useful revision activities etc.)

- $\quad$ strategies of selecting important vocabulary from a reading text

- $\quad$ some useful strategies for presenting vocabularies

- $\quad$ strategies to carry out testing and evaluation

In the second demonstration session, some participants seemed confused in handling games to carry out the activities, while some other were unable to choose appropriate activities for either presentation or practice. Still among all, the common tendency was to heed towards words containing single unit. To have a clear idea about the teachers' presentations a case study is included here.

\section{Case study:}

Like other trainee teachers this participant submitted a copy of lesson plan beforehand. The lesson plan was prepared for the students of class-vii on the basis of a selected lesson taken from English for Today. The lesson was prepared according to the steps like preparation, presentation, practice and production. Besides she collected a few posters, charts, and maps and prepared a worksheet to facilitate the learning. The class began with observations on some pictures related with the text. Then the teacher went straight to the text and read it out to the class while at the same time she circled some new vocabularies in a larger text. It should be mentioned that though the learners had texts with them, the teacher displayed a larger text on a flip board so that the learners could keep track. After finishing reading, she picked up some words and asked their meaning one by one. Some learners answered in their mother tongue and some remained quiet except for once or twice when they made correct answers in English. Each time the teacher 
corrected students' faults by giving the correct answer (without inviting other learners to answer) and wrote answers on the board while the whole class followed her and copied all in their copies. Though in the word list there were words that could be presented through actions and demonstrations, the teacher did not go for trying any of them. In the meantime the learners seemed restless and tried to converse among themselves. The class continued with frequent shouts like "keep silent" made by the teacher to manage the class. Next activity was "make sentences" using the same words but the teacher handled this activity as the previous one: she asked for the answer once and if it was incorrect or partially correct she provided a correct answer and the learners copied all from the board though the examples and explanations were often more complicated than the original word/s. Then she moved towards some activities form the worksheet based on grammar.

\section{Is knowing about the strategies enough?}

The participant teachers' demonstration revealed that knowing about the strategies is not all, but the positive outcome lies in its correct applications. The teachers also reported that they rarely use any teaching aid or supporting material in their practical classroom to teach vocabulary because of their extra work load, limitations of collecting materials and the anxiety caused by lack of self confidence in handling the techniques.

Data collected from learners and teachers about vocabulary presentation and teaching techniques have discovered few aspects worth for consideration. First of all, ESL instructors must have clear knowledge about vocabulary teaching related issues. Secondly, careful attention must be given to the selection of the specific aspect of lexis that teachers need to focus on. On some occasions phrases can help learners more than the individual words in real life communication. To explain the advantages of teaching phrases Nattinger and DeCarrico (1992), says, "Learners can creatively construct sentences simply because the phrases are stored and reprocessed as whole chunks (and) this can ease frustration and develop motivation and fluency" (p.34). The same strategy could be applied for junior classes as well 
because while introducing an object, it is possible to present a few more information associated with it.

Thirdly, learners need to be freed from the boredom of tiring activities like memorizing a long word list and their meaning as the only activity of vocabulary learning. More interesting and motivating tasks need to be introduced instead.

Fourthly, a careful selection needs to be made in case of teaching vocabulary from reading passages that contain several new words. In this case students should be introduced first with words that they need to understand the core meaning of the passage. According to Folse (2008):

To make sure that student' notice new words, teachers can write them on the board for all to see. They can also keep a separate section of the board for vocabulary. . . It is not a good idea to write down every new word; instead, teachers should write down words that they think are useful to their students. . .. (and) focus on words that are relevant to their students' actual needs, which may include actual communication or passing a standardized examination. (p.16)

Fifthly, as spelling and dictation are the activities that could support vocabulary learning, a close relation needs to be maintained among related presentation, spelling, dictation and practice tasks.

While considering the strategies commonly used in Bangladesh at present for teaching or enhancing vocabulary according to the scale of the mentioned theories, it has been observed that instructors are a bit confused in handling the Meta cognitive level as most often they cannot choose the right word/s out of many as important vocabulary items. For most of them, any new word should be learnt by the learners though for doing that they get comparatively insufficient time. For a number of instructors, vocabulary means only the individual words and they keep them away from teaching phrase and compound words. In cognitive level problem occurs when the meanings of the words become more difficult or complicated than the given word. But the main problem is in the memory building level. No doubt, after a certain period temporary memory fades away and gradually all learning is 
lost if revisions are not done after regular intervals. So as attention is given to practise, memory gets permanent. In search of Memory building activities teachers need not to go for a huge planning. For this purpose simple activities like immediate repetition/drills could also serve a lot. But teachers must be aware about the fact that if memory is not built, tasks that are attempted to build up vocabulary will go in vein. This happens in practice in Bangladesh. Memory building activities are left in negligence as teachers cannot manage adequate tasks for ensuring the practice. May be they are too much concerned about the offered syllabus and formal evaluation controlled by the education boards. Besides, in order to build up memory, interesting tasks, and variation in tasks should be brought. The same kind of activities bring boredom and maximize the dependence on memorization, therefore, the learners' achievement in terms of understanding remains incomplete. Therefore, in order to minimize the difficulties of presenting new vocabulary a minute planning is necessary. Teachers need to be innovative in planning such tasks. In this regard the well-known proverb "Give a lemon, get a glass of lemonade" could be the motto. Teachers should look forward to let students to use the newly learnt vocabularies in various ways like the following example.

It has been noted from the discussion with ESL instructors that while introducing certain nouns and verbs, they prefer to use pictures. If pictures are unavailable, they use dictionary definition like -

Bison: a large, wild animal like a cow with long hair.

Hatch: make a young bird, fish, insect etc. come out of an egg

Swaying: moving from one side to another

(According to the data collected from students' copies)

For the second word a picture could be alright along with the definition but for the first and the third word, the same manner of presentation is insufficient because, especially for the third word, the learners may misunderstand the action by guessing "scrolling" and "swaying" as same activity. But if these words are 
presented by putting pairs of pictures ( e.g. cow and bison) side by side and later learners are asked to write the difference that they have observed between two things or activities, their idea about the word/s get clearer and through it learners could practise the language in a compare and contrast situation.

\section{Recommendations:}

While planning a lesson with an objective of teaching vocabulary, language instructors need to be concerned with the following issues that indirectly facilitate learning:

- New vocabulary should be introduced along with some other words that students already know so that the learners become motivated and feel self confident.

- Repetition is necessary for learning and to reinforce the learned vocabulary. In junior classes drilling could be used as a means of pronunciation practice though towards adult learners this task often seem tiring ,however, the task that require to reuse the vocabulary that could be used instead.

- Learners should be involved in activities like conversation, letter or postcard writing, narrate or describe things or happenings etc. when they will be influenced to use the newly learnt vocabulary.

- "Indeed many learners favour the type of vocabulary books that list words frequently appearing in the . . . examination." (Lee, 2004, Para.1). Learning vocabulary from these kind of books are not a problem but problem occurs when the learners memorize words without understanding, and therefore, could use them (words) only on limited occasions or when they least bother with other words associated with those listed vocabularies in their text books and as a result remain far away from obtaining the expected goal that could be achieved from the course. In fact, most of the ESL learners in a country like Bangladesh 
attend English classes for cutting a good score in the examination. Therefore, while selecting vocabulary, teachers must honour the learners' immediate need and ensure enough practice with them so that the learners may be freed from the burden of so called blind memorization.

- Senior students must be trained for forming a habit to go through the dictionary for getting information about a new word other than depending on any other available sources because dictionary is the most authentic source for getting meaning/s of any word and its pronunciation clues.

- While choosing a reading passage for vocabulary building, teachers must make sure that the passage is not too lengthy and the number of new vocabularies must be limited. To get a good response from the learners, vocabulary learning and practising activities must be interlinked.

- Teachers must make sure that they have included pronunciation and spelling test as a regular activity of language classes as learners are found very weak in both of the cases.

\section{Conclusion:}

No doubt, learning new vocabulary and acquiring the ability to use them appropriately is a challenge for the ESL learners and achieving communicative competence without learning vocabulary is next to impossible. This study has revealed some limitations that lie in the process of teaching (including selecting, planning, training etc.) and learning (including lack of adaptation, controlling anxiety etc.) vocabulary. These limitations also cause problematic or incomplete learning and ultimately hamper learners' communicative competence growth. In fact, determining the useful vocabulary, planning a suitable lesson to carry on the activities and training the learners with suitable or helpful strategies are the principle issues to assist the learners both for achieving self motivation and acquiring a handsome word stock needed for successful communication. 


\section{Works Cited}

Cobb, T., \& Morris, L. (2004). “Vocabulary profiles as predictors of the academic performance of TESL trainees." System, 32, 75-87.

Dong, L. Q. (2009). Beyond Reading: Vocabulary expansion and language use consolidation. $5^{\text {th }}$ National VTTN ELT Conference. Retrieved from www.britishcouncil.org/lam_quang_dong.doc .

Ellis, R. (1994). The study of second language acquisition. Oxford : Oxford University Press.

Fallahzadeh, M. H. (2007). “A Survey of the students and interns' EFL writing problems in Shiraz University of Medical Science." Asian EFL Journal: English Language Teaching and Research Articles, 9(3). Retrieved from Asian EFL Journal database.

Folse, K.S. (2008). "Six vocabulary activities for the English language classroom." English Teaching Forum, 46(3), 12-20.

Ghazal, L. (2004). “Learning vocabulary in EFL contexts through vocabulary learning strategies." Novitas-Royal, 1(2), 84-91. Retrieved from www.novitasroyal.org/welcome1_2.htm .

Gnoinska, A. (1998). “Teaching vocabulary in colour.” English Teaching Forum, 36(3), 12-15.

Gu, Y., \& Johnson, R. K. (1996). “Vocabulary learning strategies and language learning outcomes." Language Learning, 46(4), 643-79.

Lee, S. (2004). “Teaching lexis to EFL students: A review of current perspectives and methods." Annual Review of Education, Communication and Language Sciences, 1. Retrieved from research.ncl. ac.uk/ARECLS/vol1_documents/Seowon/Seowon.htm.

Nation, P. (2001). Learning vocabulary in another Language, Cambridge: Cambridge 
University Press.

Nattinger, J . \& DeCarrico, J. (1992). Lexical phrases and language teaching. Oxford: Oxford University Press.

Richards, J. C. (1985). The context of language teaching. Cambridge: Cambridge University Press.

Stevik, E. W. (1976). Memory, meaning and method. Massachusetts: Newsbury House Publishers.

\section{References}

Choudhury, A.S. (2004). “Teaching vocabulary in the ESL/EFL classroom: Central pedagogical issues." Modern Journal of Applied Linguistics, 2(4) .Retrieved from $\leq$ mjal.org/journal-current-articles.htm $\geq$.

Darn, S. (2008). ELT teaching and methodology. GME: Emotional intelligence and ELT. Retrieved from http://www.teachingenglish.org.uk/think/articles/emotionalintelligence-elt

Harmon, J.M., \& Wood, Karen D. (2008). Vocabulary teaching learning across disciplines. Research Summary. Retrieved from http://www.nmsa.org/ Research/ResearchSummaries/VocabularyTeaching/tabid/1728/Default.aspx. 